\title{
Superconductivity and superconducting order parameter phase fluctuations in a weakly doped antiferromagnet
}

\author{
V.M. Loktev* \\ Bogolyubov Institute for Theoretical Physics, \\ Metrologicheskaya str. 14-b, Kiev-143, 03143 Ukraine \\ V.M. Turkowskit \\ Department of Physics and Astronomy, \\ University of Missouri-Columbia, Columbia, Missouri 65211
}

(Dated: October 4, 2018)

\begin{abstract}
The superconducting properties of a recently proposed phenomenological model for a weakly doped antiferromagnet are analyzed, taking into account fluctuations of the phase of the order parameter. In this model, we assume that the doped charge carriers can't move out of the antiferromagnetic sublattice they were introduced. This case corresponds to the free carrier spectra with the maximum at $\mathbf{k}=( \pm \pi / 2, \pm \pi / 2)$, as it was observed in ARPES experiments in some of the cuprates in the insulating state [1]. The doping dependence of the superconducting gap and the temperature-carrier density phase diagram of the model are studied in the case of the $d_{x^{2}-y^{2}}$ pairing symmetry and different values of the effective coupling. A possible relevance of the results to the experiments on high-temperature superconductors is discussed.

PACS numbers: 74.20.-z,74.20.Fg, 74.20.Rp, 74.72.-h
\end{abstract}

*Electronic address: vloktev@bitp.kiev.ua

${ }^{\dagger}$ Electronic address: turkowskiv@missouri.edu 


\section{INTRODUCTION}

Theoretical description of high-temperature superconductors (HTSCs) remains one of the most important unsolved problems of modern condensed matter theory. However, despite the lack of complete understanding of this phenomena, some facts about the cuprate materials are almost generally accepted by scientific community. First of all, it is believed that superconductivity in this materials is mainly generated in $\mathrm{CuO}_{2}$ layers, since even samples with the layer spacing of $\sim 10 \AA$ demonstrate very high critical temperatures. Second, these materials in undoped regime are antiferromagnetic insulators, which transform into superconductors when the doping is larger than some critical value. Experiments indicate the presence of strong antiferromagnetic correlations in the superconducting regime [2, 3]. Third, the symmetry of the superconducting order parameter is of a $d$-wave type in many of the compounds [4, 5]. It is believed by many researches that these facts contain sufficient information to understand the phenomena of HTSC. It is almost generally accepted that this phenomena can be described in the framework of a simple two-dimensional model of strongly correlated electrons (for a recent over-review, see [6]). The antiferromagnetic correlations produce a BCS-type spin-wave pairing between electrons. One of the most often studied model of HTSCs is the t - J-model [7, 8] (see, for example, [9, 10, 11, 12, 13, 14]). It was shown that the solution of this model within different approximations demonstrates $d$-wave superconductivity in the underdoped regime. This and many other models, however, are rather oversimplified, and they do not take into account some properties of the cuprates. In particular, it is known from ARPES experiments that the free carrier spectra of some cuprates like $\mathrm{Sr}_{2} \mathrm{CuO}_{2} \mathrm{Cl}_{2}$ in the insulating phase has its maxima not at the Brillouin zone edge at momenta $\mathbf{k}=( \pm \pi, \pm \pi)$, but at points $\mathbf{k}=( \pm \pi / 2, \pm \pi / 2)[1]$.

This fact suggests that the free carrier spectra must be $\varepsilon(\mathbf{k}) \sim-4 t_{n n} \cos k_{x} \cos k_{y}-$ $2 t_{n n n}\left(\cos 2 k_{x}+\cos 2 k_{y}\right)$, i.e. the doped carriers move only within one sublattice where they were born. This situation is similar to the case of collinear antiferromagnetic dielectrics with the Neel ground state, where electronic and spin excitations can't move out of one sublattice to another. It is important that the excitations must live on non-equivalent sublattices, i.e. on the copper sublattices, which form an antiferromagnet. It is often assumed for several reasons (see below), that doped holes in cuprates occupy sites in the oxygen subsystem of

the $\mathrm{CuO}_{2}$ planes [15]. However, it is known that the n.n.n. hopping parameter for this 
sublattice is much smaller than the n.n. hopping parameter, which excludes the possibility to explain the spectra experiments [1] in this case. One can try to overcome this difficulty by introducing the Zhang-Rice singlet, which consists of a doped oxygen hole coupled to one of the closest copper ions. The hole chooses the ion in such a way that the energy of the singlet is minimal. Such states can move only within their magnetic sublattices. However, these states are unstable due to several reasons. First of all, the "up" and "down" spins of the copper ions enter in the linear combination of the Zhang-Rice singlet in an equivalent way, so a hole with the opposite spin projection will try to form the same singlet with the neighboring localized ion spin from another sublattice. Also, the oxygen ions occupy states which are symmetrical with respect to the neighbor copper ions. Therefore, the total exchange field which acts on a hole on the oxygen site is compensated, and the hole becomes frustrated with respect to the choice of the axis of the spin quantization. The proper axis of quantization can be established, but in this case the hole hybridized with the copper states will move in the $\mathrm{CuO}_{2}$ plane not feeling the magnetic ordering. This also doesn't allow one to explain the spectra experiments.

These difficulties can be avoided by assuming that the doped holes sit on the copper sites 16]. In fact, in the undoped state the $\mathrm{CuO}_{2}$ planes consist of ions $\mathrm{Cu}^{2+}$ and $\mathrm{O}^{2-}$. The doping leads to the valence change of some ions, which results in appearance of movable charges in the system. The assumption that the holes occupy the copper ions corresponds to the transitions $\mathrm{Cu}^{2+} \rightarrow \mathrm{Cu}^{3+}$, which are different from usually considered transitions $\mathrm{O}^{2-} \rightarrow \mathrm{O}^{-}$in the case of the holes which occupy the oxygen sites. The reason for the last assumption follows from the fact that it is believed by many researches that the configuration $\mathrm{Cu}^{3+} \mathrm{O}^{2-}$ has higher energy comparing to $\mathrm{Cu}^{2+} \mathrm{O}^{-}$. However, the configuration $\mathrm{Cu}^{3+} \mathrm{O}^{2-}$ can be preferable if one takes into account the Coulomb attraction $\mathrm{V}_{\mathrm{C}}$ inside the configurations. In fact, since $\mathrm{V}_{\mathrm{C}}^{\mathrm{Cu}^{3+} \mathrm{O}^{2-}} \simeq 3 \mathrm{~V}_{\mathrm{C}}^{\mathrm{Cu}^{2+} \mathrm{O}^{-}} \simeq 6 \mathrm{~V}_{\mathrm{C}}^{\mathrm{Cu}^{+} \mathrm{O}^{-}}$, and every copper ion is surrounded by four oxygen ions, at the same time as every oxygen is surrounded by only two copper ions, one can find that the corresponding Coulomb attraction energy difference for two configurations is of order $20 \mathrm{~V}_{\mathrm{C}}^{\mathrm{Cu}^{+} \mathrm{O}^{-}}$. This difference in the Coulomb interaction energies can provide stability of the $\mathrm{Cu}^{3+} \mathrm{O}^{2-}$ configuration comparing to other configurations, i.e. a possibility for the doped holes to move within the copper sublattices. This picture corresponds qualitatively to the $\mathrm{t}-\mathrm{J}$-model case. However, it was usually assumed that the free carrier dispersion relation in this model corresponds to the nearest neighbor hopping, which does not allow 
one to obtain the experimental spectra with the minimum at $\mathbf{k}=( \pm \pi / 2, \pm \pi / 2)$.

A simple phenomenological Hamiltonian, which corresponds to charge carriers that move within fixed sublattices, was proposed in order to describe superconductivity in cuprates at low carrier densities in [16]. The effective attraction between the doped electrons on different sublattices was assumed to be equal to the antiferromagnetic coupling $J$ between nearest site spins. In fact, the carrier doping in the antiferromagnet leads to a breakdown of the antiferromagnetic coupling $J$ between nearest site spins. This results in an increasing of the energy of the system. This energy increasing is minimal when two empty sites are the nearest neighbors, because the minimal number of the antiferromagnetic spin-spin (exchange) interaction bonds is broken in this case. This phenomenological attraction was introduced for the first time by Trugman in Ref.[18]. As it was shown in Ref. [19], the order parameter, which corresponds to the pairs produced by such an interaction, has a $d$-wave symmetry.

Below, the doping dependence of the $d$-wave superconducting gap in the model at different values of coupling and $T=0$ is studied. In addition, we analyze the temperature-doping phase diagram of the model. It is shown that the width of the pseudogap temperature

region between the mean-field critical temperature $T_{c}^{M F}$ and the critical temperature of the Berezinskii-Kosterlitz-Thouless $(\mathrm{BKT})$ transition $T_{c}\left(\equiv T_{B K T}\right)$ strongly depends on the carrier concentration. We make a qualitative comparison of the results with the experimental results on some HTSCs.

\section{MODEL}

The Hamiltonian of non-interacting doped d-hole carriers in HTSCs can be approximated by

$$
H_{d}=\varepsilon_{d} \sum_{\mathbf{n}} \sum_{\sigma_{\mathbf{n}}} d_{\mathbf{n} \sigma_{\mathbf{n}}}^{\dagger} d_{\mathbf{n} \sigma_{\mathbf{n}}}-\frac{1}{2} \sum_{\mathbf{n}, \mathbf{m}} \sum_{\sigma_{n}, \sigma_{m}} t_{\mathbf{n} \mathbf{m}}\left\langle\sigma_{\mathbf{n}} \mid \sigma_{\mathbf{m}}\right\rangle d_{\mathbf{n} \sigma_{n}}^{\dagger} d_{\mathbf{m} \sigma_{m}}
$$

where $d_{\mathbf{n} \sigma_{n}}^{\dagger}\left(d_{\mathbf{n} \sigma_{n}}\right)$ is the creation (annihilation) operator of the electron on site $\mathbf{n}$ with spin $\sigma_{\mathbf{n}}, \varepsilon_{d}$ is the electron on-site energy, $t_{\mathbf{n m}}$ is the hopping parameter and $\left\langle\sigma_{\mathbf{n}} \mid \sigma_{\mathbf{m}}\right\rangle$ is spin-spin correlation function calculated in the ion system of coordinates (see 16]). This Hamiltonian can be transformed to the following form in terms of the Hubbard operators in the laboratory system of spin coordinates:

$$
H_{d}=H_{c o h}+H_{i n t}^{(1)}+H_{i n t}^{(2)}
$$


where

$$
H_{c o h}=\left(\varepsilon_{d}-\mu\right) \sum_{\mathbf{n}} X_{\mathbf{n}}^{2,2}-\frac{1}{2} \sum_{\mathbf{n}, \mathbf{m}} t_{\mathbf{n m}} \cos \frac{\mathbf{Q}_{A F M}(\mathbf{n}-\mathbf{m})}{2} X_{\mathbf{n}}^{2,1 / 2} X_{\mathbf{m}}^{1 / 2,2}
$$

is a part of the Hamiltonian which describes motion of free holes in an antiferromagnetically ordered medium, $\mathbf{Q}_{A F M} \mathbf{a}=( \pm \pi, \pm \pi)$, $\mathbf{a}$ is the lattice constant of a square lattice,

$$
H_{\text {int }}^{(1)}=-\frac{1}{2} \sum_{\mathbf{n}, \mathbf{m}} t_{\mathbf{n m}} \sin \frac{\mathbf{Q}_{A F M}(\mathbf{n}-\mathbf{m})}{2}\left(X_{\mathbf{n}}^{2,1 / 2} X_{\mathbf{m}}^{1 / 2,2} S_{\mathbf{m}}^{-}-X_{\mathbf{n}}^{2,1 / 2} X_{\mathbf{m}}^{1 / 2,2} S_{\mathbf{n}}^{+}\right)
$$

and

$$
H_{\text {int }}^{(2)}=-\frac{1}{2} \sum_{\mathbf{n}, \mathbf{m}} t_{\mathbf{n m}} \cos \frac{\mathbf{Q}_{A F M}(\mathbf{n}-\mathbf{m})}{2} X_{\mathbf{n}}^{2,1 / 2} X_{\mathbf{m}}^{1 / 2,2} S_{\mathbf{n}}^{+} S_{\mathbf{m}}^{-}
$$

describe non-coherent inter-ion hole transitions with one and two spin excitations, correspondingly. $S_{\mathbf{n}}^{+}$and $S_{\mathbf{m}}^{-}$are spin creation and annihilation operators. It is important that expressions (2)-(5) are written in terms of the Hubbard operators, which directly take into account the antiferromagnetic ordering in the system. The ion spin projections in both magnetic sublattices are equal to $1 / 2$ in the ground state of the crystal (we use local systems of coordinates for each sublattice). The spin conservation is also taken into account, which results in the fact that the electrons can move only on the magnetic sublattice on which they were born (for details, see [16]).

As it was mentioned in the Introduction, a simple effective attraction between the doped electrons on different sublattices can be introduced [18]. The carrier doping in the antiferromagnet leads to an increasing of the energy of the system, since it breaks the antiferromagnetic coupling $J$ between nearest site spins. The energy increasing is minimal when two doped particles occupy the nearest neighbor sites, since the minimal number of the antiferromagnetic spin-spin bonds is broken in this case. Therefore, the doping leads to an effective attraction between carriers on different sublattices:

$$
H_{a t t r}=-J \sum_{\mathbf{n}, \rho=\mathbf{a}, \mathbf{b}} X_{\mathbf{n}}^{2,2} X_{\mathbf{n}+\rho}^{2,2}
$$

The total Hamiltonian of the system is $H=H_{d}+H_{\text {attr }}$. For simplicity, we neglect terms (44) and (5), which do not contribute to a significant increasing of the superconducting critical temperature. The first term corresponds to a BCS-like interaction, which is small at low carrier densities, or Fermi momenta of doped holes $k_{F}$. It is proportional to the 
energy of spin waves $\Omega_{A F M}\left(k_{F}\right)$, which is much smaller than the exchange energy $J$. The term Eq. (5), which corresponds to two-magnon attraction, also doesn't contribute to a significant increasing of the critical temperature in the $d$-wave pairing channel [17].

Therefore, a simplified version of the Hamiltonian for the doped the antiferromagnet can be written as:

$$
H=\left(\varepsilon_{d}-\mu\right) \sum_{\mathbf{n}} X_{\mathbf{n}}^{2,2}-\frac{1}{2} \sum_{\mathbf{n}, \mathbf{m}} t_{\mathbf{n m}} \cos \frac{\mathbf{Q}_{A F M}(\mathbf{n}-\mathbf{m})}{2} X_{\mathbf{n}}^{2,1 / 2} X_{\mathbf{m}}^{1 / 2,2}-J \sum_{\mathbf{n}, \rho=\mathbf{a}, \mathbf{b}} X_{\mathbf{n}}^{2,2} X_{\mathbf{n}+\rho}^{2,2} .
$$

In the case of an antiferromagnet on a square lattice with two sublattices, the free particle energy spectrum, which corresponds to the first two terms in Eq. (7), is:

$$
\varepsilon(\mathbf{k})=\varepsilon_{d}-4 t_{2} \cos k_{x} \cos k_{y}-2 t_{3}\left(\cos 2 k_{x}+\cos 2 k_{y}\right)-\mu
$$

where $\mu$ is the chemical potential, and $t_{2}$ and $t_{3}$ are the next nearest and next next nearest neighbor hopping parameters, correspondingly. We use the units where the lattice constant is equal to one: $a=1$. In order to have $\varepsilon(\mathbf{k})=0$ at $\mathbf{k}=0$ one can choose $\varepsilon_{d}=4 t_{2}+4 t_{3}$. The chemical potential is connected with the free (or doped) particle number in the system by the following natural relation:

$$
\delta=\sum_{\mathbf{n}}\left\langle X_{\mathbf{n}}^{2,2}\right\rangle
$$

where the sum goes over two sublattices. The Hamiltonian Eq. (7) has a simpler structure, comparing to the t-J-model Hamiltonian, yet it can describe some of the main physical properties of underdoped cuprates.

\section{ZERO-TEMPERATURE PROPERTIES}

To study superconducting properties of the system described by the Hamiltonian Eq. (17), it is convenient to introduce generalized Nambu-Hubbard hole operators:

$$
\Psi_{\mathbf{n}}(t)=\left(\begin{array}{c}
X_{\mathbf{n}}^{2,1 / 2}(t) \\
X_{\mathbf{n}}^{1 / 2,2}(t)
\end{array}\right), \quad \Psi_{\mathbf{n}}^{\dagger}(t)=\left(X_{\mathbf{n}}^{1 / 2,2}(t), X_{\mathbf{n}}^{2,1 / 2}(t)\right)
$$

where $\mathbf{n}$ are lattice sites and $t$ is time. In this case the time ordered Green function $\hat{G}_{\mathbf{n m}}\left(t, t^{\prime}\right)=-i\left\langle T\left(\Psi_{\mathbf{n}}(t) \Psi_{\mathbf{m}}^{\dagger}\left(t^{\prime}\right)\right\rangle\right.$ is

$$
\hat{G}_{\mathbf{n m}}\left(t, t^{\prime}\right)=-i\left(\begin{array}{cc}
\left\langle T X_{\mathbf{n}}^{2,1 / 2}(t) X_{\mathbf{m}}^{1 / 2,2}\left(t^{\prime}\right)\right\rangle & \left\langle T X_{\mathbf{n}}^{2,1 / 2}(t) X_{\mathbf{m}}^{2,1 / 2}\left(t^{\prime}\right)\right\rangle \\
\left\langle T X_{\mathbf{n}}^{1 / 2,2}(t) X_{\mathbf{m}}^{1 / 2,2}\left(t^{\prime}\right)\right\rangle & \left\langle T X_{\mathbf{n}}^{1 / 2,2}(t) X_{\mathbf{m}}^{2,1 / 2}\left(t^{\prime}\right)\right\rangle
\end{array}\right) .
$$


The Green function Eq. (11) satisfies the following equation of motion:

$$
i \frac{\partial}{\partial t} \hat{G}_{\mathbf{n m}}\left(t, t^{\prime}\right)=\delta\left(t-t^{\prime}\right) \delta_{\mathbf{n m}} \hat{I}+\left\langle T\left[\Psi_{\mathbf{n}}(t), H\right] \Psi_{\mathbf{m}}^{\dagger}\left(t^{\prime}\right)\right\rangle
$$

where, as it was mentioned above, $H$ is defined by (17), and

$$
\hat{I}=\left(\begin{array}{cc}
\left\langle X_{\mathbf{n}}^{1 / 2,1 / 2}(t)+X_{\mathbf{n}}^{2,2}(t)\right\rangle & 0 \\
0 & \left\langle X_{\mathbf{n}}^{1 / 2,1 / 2}+X_{\mathbf{n}}^{2,2}\right\rangle
\end{array}\right) .
$$

This equality can be derived by using the commutation relations for the Hubbard operators.

In the generalized mean-field theory approximation, the last term in Eq. (12) can be linearized in the following way (see, for example Ref. [20]):

$$
\left\langle T\left[\Psi_{\mathbf{n}}, H\right] \Psi_{\mathbf{m}}^{\dagger}\right\rangle(\omega) \simeq \sum_{\mathbf{l}} \hat{E}_{\mathbf{n l}} \hat{G}_{\mathbf{l m}}(\omega)
$$

where

$$
\hat{E}_{\mathbf{n m}}=\left\langle\left\{\left[\Psi_{\mathbf{n}}, H\right], \Psi_{\mathbf{m}}^{\dagger}\right\}\right\rangle
$$

is the energy matrix. The nonlinear (dynamical) corrections to the self-energy in Eq. (14) can be taken into account [12]. We assume that the generalized mean-field approximation Eq. (14) is good enough in the case of low carrier densities, when the free quasi-particle excitations correspond to the field represented by the Hubbard operators Eq. (10).

The expression for the energy matrix (15) can be found by solving the Heisenberg equations of motion for the $X$-operators. In terms of the energy matrix Eq. (15), the Green function can be written as:

$$
\hat{G}_{\mathbf{n m}}(\omega)=\frac{\hat{I}}{\omega \delta_{\mathbf{n m}}-\hat{E}_{\mathbf{n m}}} .
$$

To find the explicit expression for the Green function Eq. (16), let us write down the equations of motion for the Hubbard operators:

$$
\begin{aligned}
i \hbar \frac{\partial}{\partial t} X_{\mathbf{n}}^{1 / 2,2}(t)= & \left(\varepsilon_{d}-\mu\right) X_{\mathbf{n}}^{1 / 2,2} \\
& -\frac{1}{2} \sum_{\mathbf{l}} t_{\mathbf{n} \mathbf{l}} \cos \frac{Q_{A F M}(\mathbf{n}-\mathbf{l})}{2}\left(X_{\mathbf{n}}^{1 / 2,1 / 2}+X_{\mathbf{n}}^{2,2}\right) X_{\mathbf{l}}^{1 / 2,2}-2 J \sum_{\rho} X_{\mathbf{n}+\rho}^{2,2} X_{\mathbf{n}}^{1 / 2,2}, \\
i \hbar \frac{\partial}{\partial t} X_{\mathbf{n}}^{2,1 / 2}(t)= & -\left(\varepsilon_{d}-\mu\right) X_{\mathbf{n}}^{2,1 / 2} \\
& +\frac{1}{2} \sum_{\mathbf{l}} t_{\mathbf{l n}} \cos \frac{Q_{A F M}(\mathbf{l}-\mathbf{n})}{2} X_{\mathbf{l}}^{2,1 / 2}\left(X_{\mathbf{n}}^{1 / 2,1 / 2}+X_{\mathbf{n}}^{2,2}\right)+2 J \sum_{\rho} X_{\mathbf{n}}^{2,1 / 2} X_{\mathbf{n}+\rho}^{2,2} .
\end{aligned}
$$


Substitution of the expressions Eqs. (17) and (18) instead of the anti-commutators $(\mathrm{i} d X / d t=[X, H])$ into Eq. (15) and evaluation of the anti-commutators give the following expression for the energy matrix:

$$
\hat{E}_{\mathbf{n m}}=\tilde{E}_{\mathbf{n m}} \hat{\tau}_{z}+\tilde{\Delta}_{\mathbf{n m}} \hat{\tau}_{x}
$$

where $\hat{\tau}_{x}$ and $\hat{\tau}_{z}$ are the Pauli matrices and

$$
\begin{aligned}
\tilde{E}_{\mathbf{n m}}= & -\delta_{\mathbf{n m}}\left(\varepsilon_{d}-\mu\right)\left\langle X_{\mathbf{n}}^{1 / 2,1 / 2}+X_{\mathbf{n}}^{2,2}\right\rangle+\frac{1}{2} \delta_{\mathbf{n m}} \sum_{\mathbf{l}} t_{\mathbf{l n}} \cos \frac{\mathbf{Q}_{A F M}(\mathbf{l}-\mathbf{n})}{2}\left\langle X_{\mathbf{l}}^{2,1 / 2} X_{\mathbf{n}}^{1 / 2,2}\right\rangle \\
& \left.+2 \delta_{\mathbf{n m}} \sum_{\mathbf{l}} J_{\mathbf{n l}}\left\langle X_{\mathbf{n}}^{1 / 2,1 / 2} X_{\mathbf{l}}^{2,2}\right\rangle+\frac{1}{2} t_{\mathbf{m n}} \cos \frac{\mathbf{Q}_{A F M}(\mathbf{m}-\mathbf{n})}{2}\left\langle X_{\mathbf{m}}^{1 / 2,1 / 2}\left(X_{\mathbf{n}}^{1 / 2,1 / 2}\right)+X_{\mathbf{n}}^{2,2}\right)\right\rangle, \\
& \tilde{\Delta}_{\mathbf{n m}}=-\frac{1}{2} \delta_{\mathbf{n m}} \sum_{\mathbf{l}} t_{\mathbf{l n}} \cos \frac{\mathbf{Q}_{A F M}(\mathbf{l}-\mathbf{n})}{2}\left\langle X_{\mathbf{l}}^{2,1 / 2} X_{\mathbf{n}}^{2,1 / 2}\right\rangle-2 J_{\mathbf{n m}}\left\langle X_{\mathbf{n}}^{2,1 / 2} X_{\mathbf{m}}^{2,1 / 2}\right\rangle .
\end{aligned}
$$

are the renormalized energy and the superconducting gap matrices. Despite their complicated formal structure, it is possible to show that the terms in Eq. (20) and (21) have a very simple physical interpretation. In particular, the second and the third terms in Eq. (20), proportional to $\delta$, lead to a renormalization of the chemical potential $\mu \rightarrow \mu^{\prime}=\mu+\delta \mu$. The average $\left.\left\langle X_{\mathbf{m}}^{1 / 2,1 / 2}\left(X_{\mathbf{n}}^{1 / 2,1 / 2}\right)+X_{\mathbf{n}}^{2,2}\right)\right\rangle$ multiplied by the hopping operator $t_{\mathbf{m n}}$ in the last term of Eq. (20) leads to renormalization of the quasiparticle bandwidth in the limit of low doping. However, one can put $\left.\left\langle X_{\mathbf{m}}^{1 / 2,1 / 2}\left(X_{\mathbf{n}}^{1 / 2,1 / 2}\right)+X_{\mathbf{n}}^{2,2}\right)\right\rangle \simeq 1$ in this limit, since in the limit of low carrier concentrations $\left.X_{\mathbf{n}}^{1 / 2,1 / 2}\right)+X_{\mathbf{n}}^{2,2} \simeq 1$, and the renormalization of the quasiparticle band is not strong. Therefore, the energy function Eq. (20) can be approximated by the free energy spectra expression Eq. (8) multiplied by -1 in the momentum space. It is also assumed that the chemical potential in Eq. (8) is renormalized.

The expression for the gap function Eq. (21) can be also simplified. In fact, as it was shown in Ref. [19], the attraction (6) favours a superconducting pairing with the $d$-wave symmetry of the order parameter. Therefore, we assume that the strongest pairings in the system takes place in the $d$-wave pairing case, and neglect the first term in Eq. (21), which doesn't contribute to the pairing in the d-wave channel. Therefore, the gap function can be approximated in the following way in the momentum representation:

$$
\Delta(\mathbf{k})=-2 \sum_{\mathbf{q}} J(\mathbf{k}-\mathbf{q})\left\langle X_{-\mathbf{q}}^{2,1 / 2} X_{\mathbf{q}}^{2,1 / 2}\right\rangle,
$$


where we introduced the nearest neighbor attraction kernel $J(\mathbf{k})=$ $2 J\left[\cos \left(k_{x}-q_{x}\right)+\cos \left(k_{y}-q_{y}\right)\right]$. Thus, Green function (16) has the following form in the momentum space:

$$
G(\omega, \mathbf{k})=\frac{1}{\omega+\varepsilon(\mathbf{k}) \tau_{z}-\Delta(\mathbf{k}) \tau_{x}}
$$

where we assumed that in the limit of low carrier densities the normalization matrix is approximately equal to the unit matrix $\hat{I} \simeq \hat{1}$.

To find the unknown gap function $\Delta(\mathbf{k})$ and the renormalized chemical potential $\mu^{\prime}$, one can write down and solve the system of equations for these functions by using the fluctuation-dissipation theorem:

$$
\langle A B\rangle=\frac{1}{\pi} \int_{-\infty}^{\infty} d \omega \frac{d \omega \Im G_{A B}(\omega)}{e^{(\omega-\mu) / T}+1} .
$$

The zero-temperature equations which connect $\Delta(\mathbf{k})$ and $\mu$ with the parameters $t_{2}, t_{3}, J$ and $\delta$ follow from Eqs. (22) and (9):

$$
\begin{aligned}
\Delta(\mathbf{k}) & =-2 \sum_{\mathbf{q}} J(\mathbf{k}-\mathbf{q}) \frac{\Delta(\mathbf{q})}{\sqrt{\varepsilon^{2}(\mathbf{q})+\Delta^{2}(\mathbf{q})}}, \\
\delta & =\sum_{\mathbf{k}}\left[1+\frac{\varepsilon(\mathbf{k})}{\sqrt{\varepsilon^{2}(\mathbf{k})+\Delta^{2}(\mathbf{k})}}\right] .
\end{aligned}
$$

Since we consider the case, when the pairing in the system takes place in the $d$-wave channel, we put $\Delta(\mathbf{k})=\Delta_{d} \gamma_{d}(\mathbf{k})$, where $\gamma_{d}(\mathbf{k})=\left(\cos k_{x}-\cos k_{y}\right)$, in Eqs. (25) and (26). In order to these equations in this case, one must extract the $d$-wave piece form the interaction kernel and approximate it by this function: $J(\mathbf{k}-\mathbf{q}) \rightarrow 2 J \gamma_{d}(\mathbf{k}) \gamma_{d}(\mathbf{q})$. In this case, Eq. (25) for the superconducting order parameter acquires a rather simple form

$$
1=4 J \sum_{\mathbf{q}} \gamma_{d}^{2}(\mathbf{q}) \frac{1}{\sqrt{\varepsilon^{2}(\mathbf{q})+\Delta_{d}^{2} \gamma_{d}^{2}(\mathbf{q})}} .
$$

The solution of the set of Eqs. (26) and (27) at different values of interaction and hopping $t_{3}$ is presented in Fig.1. As it follows from this Figure, the gap is not very sensitive to the values of the next-nearest neighbor hopping parameter, but it strongly depends on the interaction potential. Superconductivity is suppressed when the carrier density is smaller than some critical value. This value is also increasing when the effective attractive interaction $J$ is decreasing. This situation is, in principle, similar to the case with attracting electrons, when there is no antiferromagnetic background for the carrier motion (see, for example $[24,[25])$. 

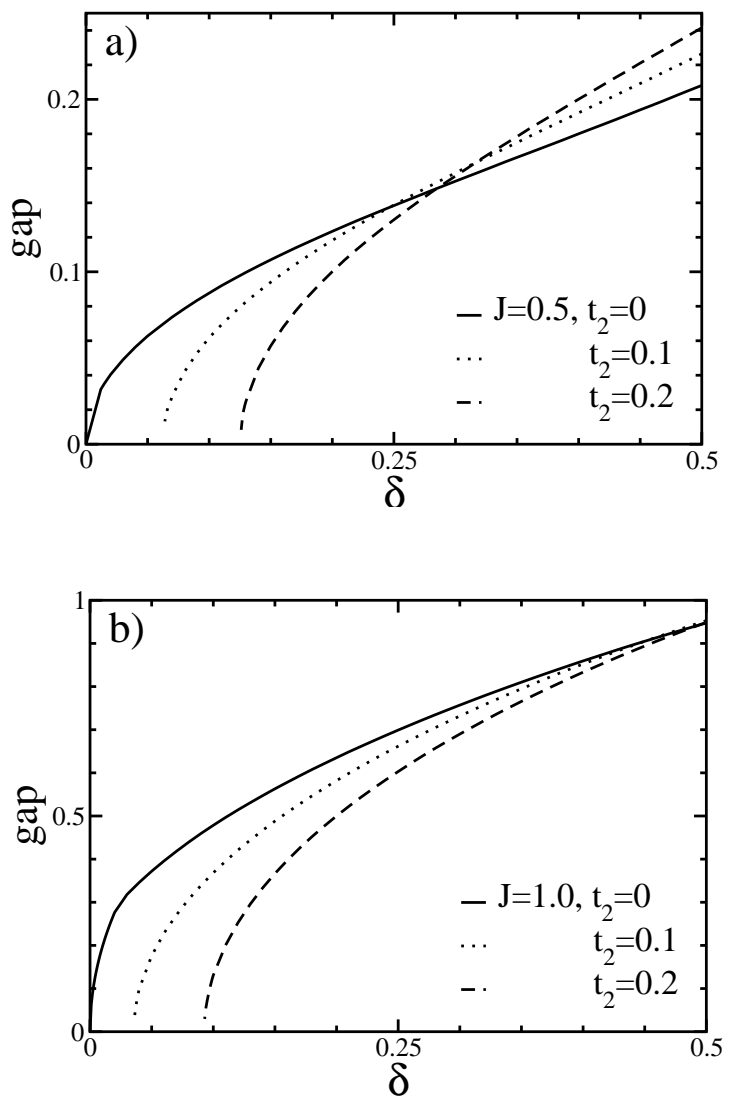

FIG. 1: The gap as function of carrier density at $t_{3}=1$, and different values of $t_{2}$ and $J$. Here and below all functions are expressed in units of $t_{3}$. Here and in Fig.2b), we don't present some results in the case when the gap or the critical temperature is very small, since it was difficult to get accurate numerical solutions in these cases.

\section{TEMPERATURE-CARRIER DENSITY PHASE DIAGRAM}

In this Section, we study the finite temperature properties of the model. It is known that there are two critical temperatures in the two-dimensional superconducting systems: the mean-field critical temperature $T_{c}^{M F}$, below which the uncorrelated pairs start to form, and the Berezinskii-Kosterlitz-Thouless critical temperature $T_{B K T}<T_{c}^{M F}$, below which the phases of the pair wave functions become algebraically ordered (for over-review, see for example [21]). Since such an order is the only possible order in our system, as it was stated above, we have to put $T_{B K T}=T_{c}$. 


\section{A. The mean-field critical temperature}

To find the dependence of the critical temperature $T_{c}^{M F}$ on the particle density in the d-wave pairing channel, we need to solve the finite-temperature version of the set of Eqs. (25) and (26). These equations follow from (24), (9) and (22):

$$
\begin{aligned}
1 & =4 J \sum_{\mathbf{q}} \gamma_{d}^{2}(\mathbf{q}) \tanh \left(\frac{\sqrt{\varepsilon^{2}(\mathbf{q})+\Delta_{d}^{2} \gamma_{d}^{2}(\mathbf{q})}}{2 T}\right) \frac{1}{\sqrt{\varepsilon^{2}(\mathbf{q})+\Delta_{d}^{2} \gamma_{d}^{2}(\mathbf{q})}}, \\
\delta & =\sum_{\mathbf{k}}\left[1+\tanh \left(\frac{\sqrt{\varepsilon^{2}(\mathbf{q})+\Delta_{d}^{2} \gamma_{d}^{2}(\mathbf{q})}}{2 T}\right) \frac{\varepsilon(\mathbf{k})}{\sqrt{\varepsilon^{2}(\mathbf{k})+\Delta_{d}^{2} \gamma_{d}^{2}(\mathbf{k})}}\right] .
\end{aligned}
$$

The system of equations for $T_{c}^{M F}$ and $\mu^{\prime}$ can be obtained from Eqs. (28) and (29) by putting the amplitude of the order parameter equal to zero. The solution of these equation shows that the doping-dependence of the mean-field critical temperature (Figs.2-4) have qualitativel.
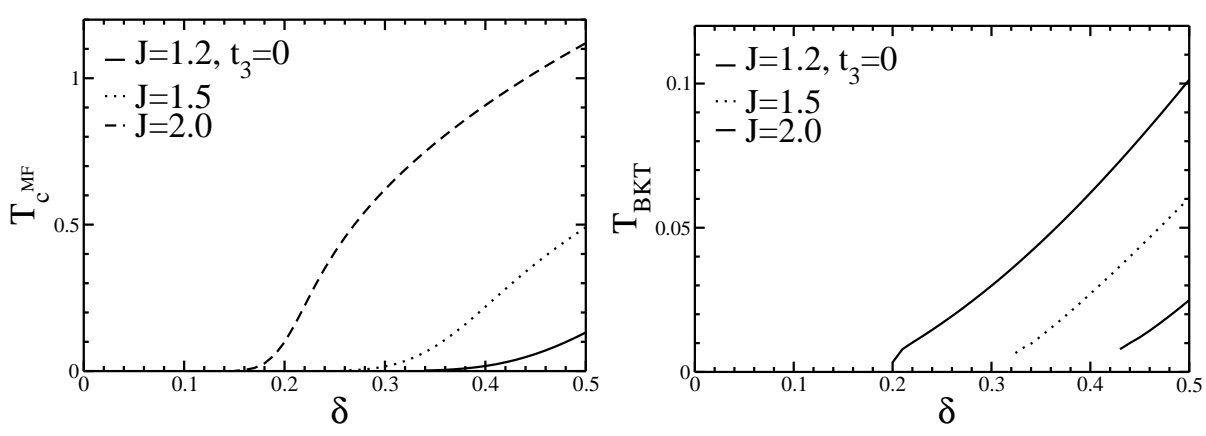

FIG. 2: The mean-field (left) and the BKT (right) critical temperatures as functions of carrier density at $t_{2}=1, t_{3}=0$ and different values of $J$.

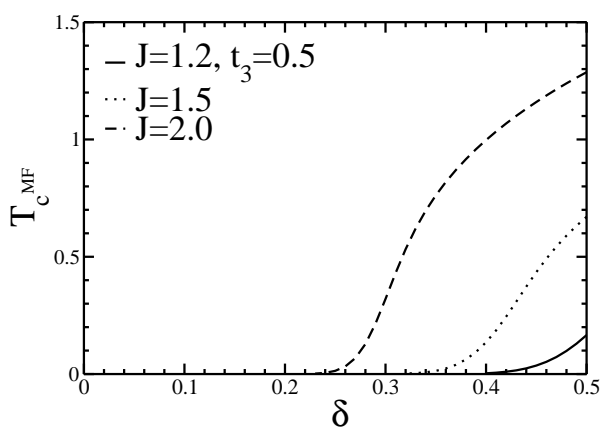

FIG. 3: The mean-field critical temperature as a function of carrier density at $t_{3}=0.5$. 


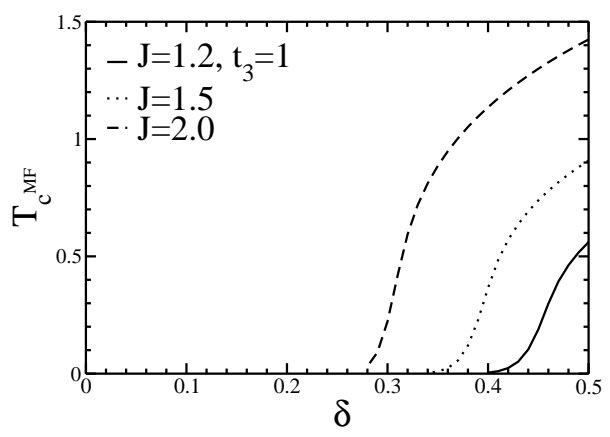

FIG. 4: The same as in Fig. 3 at $t_{3}=1$.

It is important that superconductivity is suppressed at low carrier densities in the $d$-wave pairing channel. To understand this qualitatively, one can notice that at low doping, the function $\gamma_{d}^{2}(\mathbf{q})$ in the equation for the mean-field critical temperature (28) can be approximated as $\gamma_{\mathbf{q}} \simeq q^{2} \cos \left(2 \varphi_{\mathbf{q}}\right) / 2 \sim m^{*} \epsilon_{F} \cos \left(2 \varphi_{\mathbf{q}_{F}}\right) / 2$, where $\epsilon_{F}$ is the Fermi energy and $m^{*}$ is an effective quasiparticle mass, which is directly connected with $t_{2}$ and $t_{3}$ (see the next Subsection). In this case, Eq. (27) has the form of a standard BSC equation for the critical temperature in the s-wave pairing channel with the renormalized coupling $J \rightarrow J m^{* 2} \epsilon_{F}^{2} / 4$. Therefore, the mean field critical temperature is exponentially small function of square of the carrier density $\delta^{2} \sim \epsilon_{F}^{2}$ in this case.

\section{B. The critical temperature}

In order to study the BKT transition in the system, it is necessary to consider the superconducting order parameter transfer phase fluctuations. The phase of the order parameter can be introduced in analogy with the fermion case, where the phase $\theta_{\mathbf{n m}}$ of the fermion operator can be presented as a product of the neutral operator $\chi(\tau, \mathbf{n})$ and its phase $\exp \{i \theta(\tau, \mathbf{n}) / 2\}[21]$. In our case:

$$
X_{\mathbf{n}}^{2,1 / 2}=\left(X_{\mathbf{n}}^{1 / 2,2}\right)^{\dagger}=\chi(\tau, \mathbf{n}) \exp \{i \theta(\tau, \mathbf{n}) / 2\}
$$

In this case, the superconducting order parameter can be presented as a product of its amplitude and the phase:

$$
\left\langle X_{\mathbf{n}}^{2,1 / 2} X_{\mathbf{m}}^{2,1 / 2}\right\rangle=\delta_{\mathbf{n}, \mathbf{m}+\rho} \Delta_{\mathbf{n m}} \exp \left(i \theta_{\mathbf{n m}}\right)
$$

It can be shown that the phase-dependence of the thermodynamic potential of the fermion 
system with the Green function (23) and the gap function (30) in the limit of small fluctuations of the phase of the order parameter is equal to

$$
\Omega(\Delta, \theta)=\frac{\mathcal{J}}{2} \int d^{2} r(\nabla \theta)^{2}
$$

where the stiffness in the long-wave limit is

$$
\mathcal{J}=\frac{\delta}{4 m^{*}}-\frac{1}{16 m^{* 2}} \frac{1}{T} \int \frac{d^{2} k}{(2 \pi)^{2}} \frac{\mathbf{k}^{2}}{\cosh ^{2}\left[\sqrt{\varepsilon(\mathbf{k})^{2}+\Delta_{d}^{2} \gamma_{d}^{2}(\mathbf{k})} / 2 T\right]}
$$

(see, for example [22]). In our case, the effective mass of the free quasi-particles is $m^{*}=$ $1 /\left[4\left(t_{2}+2 t_{3}\right)\right]$.

In analogy with the 2D spin $X Y$-model [23], the equation for the BKT transition critical temperature, below which the phases of order parameter (the spin $\nabla \theta$ orientation in the $X Y$-model case) become algebraically ordered has the following form:

$$
T_{c}=\frac{\pi}{2} \mathcal{J}\left(\Delta_{d}, \mu^{\prime}, T_{c}\right)
$$

where function $\mathcal{J}$ is defined in (32).

The doping dependence of the superconducting critical temperature $T_{c}$ can be found by solving the system of Eqs. (28), (29) and (33). The solution of this set at different values of interaction shows that the doping-dependence of $T_{c}$ has qualitatively the same form as the doping-dependence of $T_{c}^{M F}$ (Fig.4). It is possible to study some limiting cases of the solution of Eq. (33) analytically. In particular, in the limit of rather large carrier densities, when $\Delta_{d} \ll T_{c}\left(T_{c}\right.$ is close to $\left.T_{c}^{M F}\right)$, one can make an expansion in powers of $\Delta_{d} / T_{c}$ on the right hand side of Eq. (33). In this case, this equation transforms to:

$$
T_{c}^{3}=A \Delta_{d}^{2}\left(T_{c}\right) \delta^{3} / m^{*}
$$

where $A \simeq \pi^{3} / 128$. Since the gap parameter depends on the critical temperature as $\Delta_{d}(T)=$ $\Delta_{d}(0)\left[1-\left(T / T_{c}^{M F}\right)^{2}\right]^{1 / \alpha}$, where $\alpha \geq 1$, at temperatures close to the mean-field critical temperature [24], the solution of Eq. (34) is:

$$
T_{c} \simeq T_{c}^{M F}\left[1-\frac{1}{2}\left[\frac{m^{*} T_{c}^{M F 3}}{A \Delta_{d}^{2}(0)}\right]^{\alpha / 2} \frac{1}{\delta^{3 \alpha / 2}}\right] .
$$

In other words, the critical temperature approaches to the mean-field critical temperature as the doping increases at large carrier densities. It is interesting, that equation (34) is also valid 
in the limit of low carrier concentrations when both critical temperatures are suppressed. Therefore, the second term in Eq. (32) is important at any carrier concentration in the dwave pairing channel, contrary to the s-pairing case, where this term can be omitted at low values of $\delta$ and $T_{c} \sim \delta$.

It is important that the amplitude of $T_{c}$ is much smaller than $T_{c}^{M F}$, and the pseudogap region $T_{c}<T<T_{c}^{M F}$ is rather large in this case (Fig.4). This is similar to the phase diagram of cuprates, where there is a large pseudogap region above the critical temperature at low carrier densities. It must be stressed, that in the case of higher carrier densities, one must condsider a model with more complicated effective interaction, comparing to Eq. (6) )

In order to get superconductivity to develop at $\delta \simeq 0.05$, similar to some of HTSCs, one needs to choose a pretty large interaction energy $J>5 t_{2}$, which correspond to the energy scale of $1 \mathrm{eV}$. However, to make a quantitative comparison with experiments, one needs to take into account different effects, which were omitted in this paper. In particular, optical phonons can give a significant contribution into the electron-electron interaction in cuprates, which can lead to decreasing of the required values of $J$ in order to get superconductivity at $\delta \simeq 0.05$.

\section{CONCLUSION}

To conclude, we have studied the superconducting properties of an effective model introduced in [16] in order to describe low carrier density properties of HTSCs. It was shown that the $d$-wave pairing superconductivity in this model exists when the carrier density is larger then some critical density. This critical density strongly depends on the interaction energy, and it is growing with interaction decreasing. The amplitude of the pseudogap temperature is much larger than the critical temperature values, which resembles the experimental situation on cuprates. There are some open issues which must be resolved. First of all, it is important to understand how to generalize the results on the case of larger carrier densities. In this case, the antiferromagnetic sublattice breaks down and it is not enough to put the attraction to be equal to the antiferromagnetic bond energy $J$. The effective antiferromagnetic attraction decreases. Also, the doping increasing is accompanied by increasing of the number of scattering centers created by dopants, which also leads to suppression of super-

conductivity (see, for example [24]). Another important problem which is widely discussed 
nowadays is to understand, whether the model can give an inhomogeneous superconducting state (see, e.g. [26] and references therein). It is also necessary to estimate pairings in other channels with different symmetry of the order parameter. These and some other questions are planned to be studied in the nearest future.

[1] A. Damascelli, Z. Hussain, and Z.-X. Shen, Rev. Mod. Phys. 75,473 (2003).

[2] J.P. Franck, in Physical Properties of High Temperature Superconductors IV, ed. D.M. Ginsberg, World Scientific, Singapore, P.189 (1994).

[3] Z.-X. Shen and D.S. Dessau, Phys. Rep. 253, 1 (1995).

[4] H. Ding, T. Yokota, J.C. Campuzano et al, Nature (London) 382,51 (1996).

[5] A.G. Loeser, Z.-X. Shen, D.S. Dessau, Science 273, 325 (1996).

[6] P.A. Lee, N. Nagaosa, and X.-G. Wen, Rev. Mod. Phys. 78, 17 (2006).

[7] P.W. Anderson, Science 235, 1196 (1987).

[8] F.C. Zhang and T.M. Rice, Phys. Rev. B 37, 3759 (1988).

[9] S. Schmitt-Rink, C. M. Varma, and A. E. Ruckenstein, Phys. Rev. Lett. 60, 2793 (1988).

[10] C. L. Kane, P. A. Lee, and N. Read, Phys. Rev. B 39, 6880 (1989).

[11] G. Martinez and P. Horsch, Phys. Rev. B 44, 317 (1991).

[12] N. M. Plakida and V. S. Oudovenko, Phys. Rev. B 59, 11949 (1999).

[13] D. Bensimon, R. Zeyher, preprint cond-mat/0603585.

[14] K. Haule, G. Kotliar, preprint cond-mat/0605149

[15] V.J. Emery, Phys. Rev. Lett. 58, 2794 (1987).

[16] V.M. Loktev, Fiz. Nizk. Temp. 31, 1 (2005).

[17] V.S. Babichenko, Yu. Kagan, JETP Lett. 56, 305 (1992).

[18] S.A. Trugman, Phys. Rev. B 37, 1597 (1988).

[19] D.J. Scalapino and S.A. Trugman, Philos. Mag. B 74, 607(1996).

[20] F. Mancini, A. Avella, Adv. Phys. 53, 537 (2004).

[21] V.M. Loktev, R.M. Quick, S.G. Sharapov, Phys. Rep. 349, 2 (2001).

[22] S.G. Sharapov, H. Beck, V.M. Loktev, Phys. Rev. B 64, 134519 (2001).

[23] Yu.A. Izyumov, Yu.M. Skryabin, Statistical Mechanics of Magnetically Ordered Systems, Plenum, New York (1988). 
[24] V.M. Loktev, V. Turkowski, Int. Journ. of Mod. Phys. B 18, 2035 (2004).

[25] V.M.Loktev and V.Turkowski, Physica C 383, 256 (2002).

[26] W.-F. Tsai and S. Kivelson, Phys. Rev. B 73, 214510 (2006). 